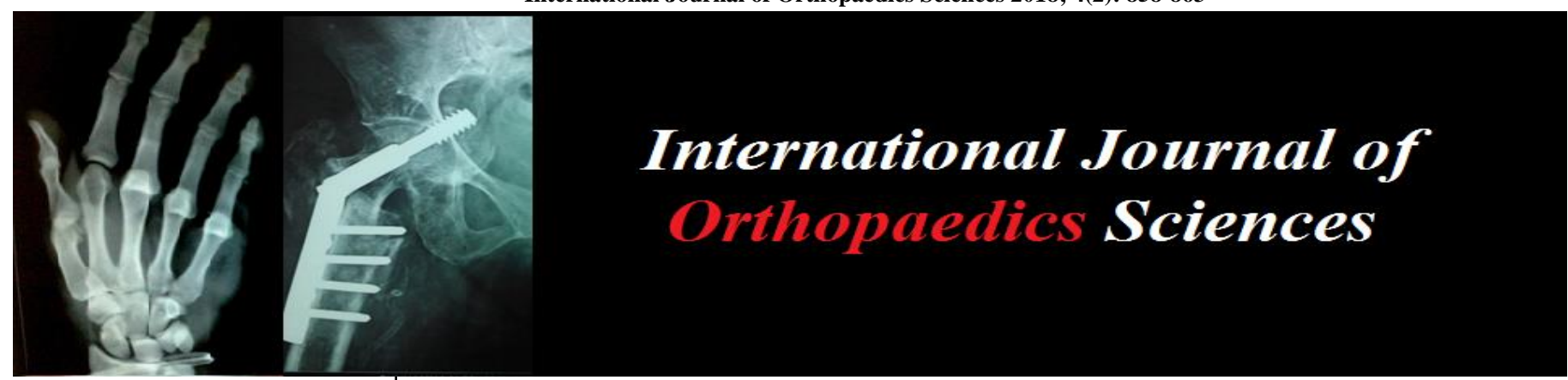

ISSN: $2395-1958$

IJOS 2018; 4(2): 858-863

(C) 2018 IJOS

www.orthopaper.com

Received: 03-02-2018

Accepted: 04-03-2018

\section{Piyush Gawai}

Department of Orthopaedics. Grant Government Medical

College and JJ Hospital,

Mumbai, Mumbai, Maharashtra,

India

Ujwal Ramteke

Assistant Professor, Department of Orthopaedics. Grant

Government Medical College and JJ Hospital, Mumbai, Mumbai,

Maharashtra, India

Sandeep Gavhale

Department of Orthopaedics.

Grant Government Medical

College and JJ Hospital,

Mumbai, Mumbai, Maharashtra,

India

\section{Harshit Dave}

Department of Orthopaedics.

Grant Government Medical

College and JJ Hospital,

Mumbai, Mumbai, Maharashtra, India

\section{Amit Yadav}

Department of Orthopaedics. Grant Government Medical College and JJ Hospital, Mumbai, Mumbai, Maharashtra, India

\section{Akash Mane}

Department of Orthopaedics. Grant Government Medical College and JJ Hospital, Mumbai, Mumbai, Maharashtra, India

\section{Vacuum assisted closure: Review on current application for post-operative wound management in orthopaedics}

\author{
Piyush Gawai, Ujwal Ramteke, Sandeep Gavhale, Harshit Dave, Amit \\ Yadav and Akash Mane
}

DOI: $\underline{\text { https://doi.org/10.22271/ortho.2018.v4.i2m.123 }}$

\begin{abstract}
Background: Post operative wound dehiscence is a significant health problem. In imposes social and financial burdens. If the injury involves the exposure of bone, early coverage of the defect must be a goal of treatment to prevent secondary problems such as an osteogenic infection. Vacuum-assisted closure (VAC) therapy has been developed as an alternative to the standard forms of wound management, which incorporates the use of negative pressure to optimise conditions for wound healing and requires fewer painful dressing changes. It is a wound management system that exposes a wound bed to local negative pressure to promote healing. This article reviews the use of VAC therapy in a post operative of wound.

Material and method: A prospective study conducted in department of orthopaedic surgery, Grant medical college and sir JJ Group of hospital, Mumbai from 2016-2017. A total of 25 subjects were consecutively recruited. Patients included in study are classified according to the grade of the ulcer. Patients age group 21-70 years with the following risk factors and comorbidities diabetes mellitus, hypertension, chronic obstructive pulmonary disease, dyslipidaemia, and smoking. Post operative wound dehiscence of proximal tibia, distal tibia and olecranon selected. Pre and post VAC culture collected. Patients were evaluated post VAC after definitive management for functional outcome.

Result: Our study of confirms that vacuum assisted closure is an excellent treatment modality for post operative wound dehiscence. Patients had an average of 8 treatment sessions. The duration of VAC application ranged from 12 to 36 days with an average hospital stay of 24 days. Amongst this maximum patients being in the age group of 30-70 years. VAC dressing found to produce more secondary closure of wound and split skin graft, also reduced more invasive procedures like flap surgery and in worst case amputation. Also it found reduces bacterial count of the wound.

Conclusion: Promotes early rehabilitation, and alleviates the need for a second procedure. Significant reduction in hospital stay subsequent lower in hospital cost, decreased amputations, increasing the number of patients undergoing skin grafting and improving culture sterility.
\end{abstract}

Keywords: VAC, Vacuum assisted closure, post-operative wound dehiscence, negative pressure wound therapy, ulcer, wound

\section{Introduction}

Post operated wound dehiscence are frequent problems in hospitalized patients, responsible for increased costs and high morbidity, demanding complex resources for their treatment Wound dehiscence is one of the most common complications of surgical ulcer, involving the breaking open of the surgical incision along the stitch. Patients with diabetes more susceptible to development of wound dehiscence following orthopaedic surgery and should be followed particularly postoperatively care.

There are several risk factors for wound dehiscence including intraoperative, postoperative factors and patient-specific. Intra operative factors include the location of the incision; the creation of large laterally based skin flaps, which have inferior blood supply; and poor softtissue handling. Also, many factors are responsible for failed wound healing, such as age, peripheral vascular disease, infection, obesity, smoking, patient's inadequate nutrition, increased pressure applied to the wound edges (generated by straining or lifting, coughing, sneezing, vomiting), corticosteroids chronic usage, previous scarring.
Ujwal Ramteke

Assistant Professor, Department of Orthopaedics. Grant

Government Medical College and JJ Hospital, Mumbai, Mumbai, Maharashtra, India 
Obesity and diabetes mellitus are significantly associated with postoperative wound-healing complications and the need for reoperation for these wound. Treatment of post operated wounds using conventional methods is frequently limited by inadequate local wound conditions, or by a poor systemic clinical situation. Patients restricted to bed, with unstable conscience level and associated diseases, present delayed formation of granulation tissue, lesser contraction of the wound, and increased local bacterial population. Healing is impaired, adding hospitalization time, causing discomfort and pain.

Table 1: Grading of wound failure ${ }^{[1]}$

\begin{tabular}{|c|c|}
\hline Grade of wound failure & Description \\
\hline Grade 0 & Simple wound erythema only without any breakdown of the skin. \\
\hline Grade I & $\begin{array}{l}\text { Skin necrosis and breakdown of the superficial wound without involvement of the deep layers or the presence of a } \\
\text { wound sinus }\end{array}$ \\
\hline Grade II & More extensive superficial necrosis associated with a wound sinus into the joint but without deep wound breakdown. \\
\hline Grade III & More extensive superficial necrosis associated with a wound sinus into the joint with deep wound breakdown. \\
\hline Grade IV & Deep dehiscence with obvious exposure of the prosthesis. \\
\hline
\end{tabular}

Vacuum assisted closure is being increasingly used for wound management. (Figure 1 and 2) Vacuum system may promote faster granulation tissue formation, remove excessive exudate, increase blood flow in the wound, and attract the borders of the wound to the centre, reducing its dimension ${ }^{[2]}$. The use of a vacuum system and hydrophobic sponge, connected to a device that produces a negative pressure on the system and over the wound. Negative pressure seems to promote drainage of excessive fluids from the wound bed and interstitial space, reducing bacterial population and oedema, and to enhance local blood flow and granulation tissue formation ${ }^{[3]}$. Other effect of vacuum is to pull of the wound borders to the centre, reducing the wound diameters due to retraction.

This mechanically induced negative pressure removes fluid from the extravascular space, improves circulation in oedematous tissue by lowering capillary afterload and enhances the proliferation of reparative granulation tissue by virtue of the ilyzarovian effect on surface reparative tissues ${ }^{[4]}$. Wounds heal by progressing through phases. After the initial injuring mechanism, an inflammatory response is brought about characterised by an array of vascular, cellular and humoral events. The traumatised tissue causes a release of vasoactive substances which, in turn, causes the capillaries to allow for the leaking of plasma proteins, followed by fluid moving from the intravascular compartment to the extravascular tissue space. The process also triggers white cell migration and enzymatic action on dead cells which, in turn, increases the osmotic load in the tissue space. This is followed by the further release of fluid from the intravascular space into the extravascular tissue space with elevation of tissue pressure and increased capillary afterload. This results in a slowing of capillary flow, decreased oxygen exchange at the tissue level and the potential for further cellular necrosis, establishing a positive feedback loop on this destructive process ${ }^{[5]}$. Once this inflammatory course and sequential events have run their course, and the necrosis is cleared, this phase gives way to a reparative phase, marked by heightened angiogenesis, granulation, collagen production and reepithelialisation. The positive effects of sub atmospheric pressure on a wound bed are many. First, the technique acts to pull fluid from the interstitial space, thereby lowering the capillary afterload in the zone of stasis. Evacuation of this fluid removes the embarrassment to the venular side of the microcirculation, enhancing flow and the delivery of oxygen and glucose to and the removal of waste products from the affected tissues [6]. Inhibitory factors that suppress the formation of fibroblasts, keratinocytes and vascular endothelial cells are removed with this fluid as well. Secondly, there is a notable increase in blood flow in the tissues of the wound ${ }^{[7]}$.

Next, it has been shown that VAC treatment lowers bacterial counts present on wound surfaces. This article reviews some of the work published so far and explains the postulated mechanisms of action of the VAC as well as its clinical applications to date in post operative wound dehiscence.

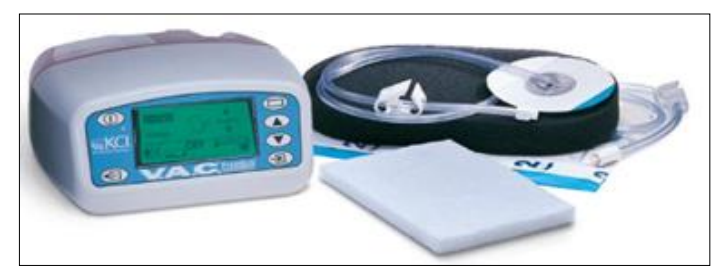

Fig 1: VAC set

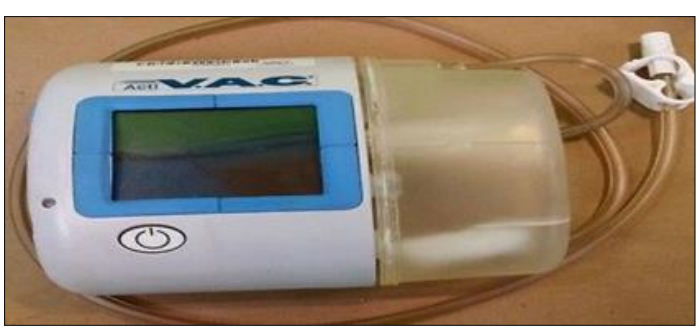

Fig 2: VAC set with canister

\section{Materials and Method}

A prospective study conducted in department of orthopaedic surgery, Grant medical college and sir JJ Group of hospital, Mumbai from 2016-2017. Informed and written consent was obtained from all patients with consent form approved by the Institutional ethical committee. A total of 25 subjects were consecutively recruited for the study randomly. Patients included in study are classified according to the grade of the ulcer (Wagner classification). All grades were included except grade 0 . Patients below 21 years and above 70 years were excluded from the study. There were 15 male and 10 female patients with the following risk factors and comorbidities diabetes mellitus, hypertension, chronic obstructive pulmonary disease, dyslipidaemia and smoking. Patients with malignancy, active bleeding and those undergoing anticoagulant therapy, gangrenous foot, exposed blood vessels, untreated osteomyelitis were excluded. Of these 12 patients presented with wound dehiscence of a proximal tibia that occurred after surgery, 10 patients presented with wound dehiscence of a distal tibia and 3 patients with wound dehiscence of olecranon fracture post operatively. Samples from all patients with surgical wound dehiscence were submitted to wound culture and specific antibiotic therapy in 
accordance with the antibiogram results was initiated. X-ray was taken to rule out active osteomyelitis. Selection criteria for VAC therapy were wound dehiscence and absence of granulation tissue 7 days after surgery. Patient selected for VAC therapy underwent wound debridement and haemostasis was achieved. Pre VAC culture was taken. A sub-atmospheric localised pressure was applied on the wound, in a controlled manner: the system unit is programmed to deliver controlled negative pressure ranging from 100 to $125 \mathrm{mmHg}$. Suction effect, generated by a portable, adjustable pump, was applied on the cleaned wound; a sponge made of reticulated polyurethane or polyvinyl alcohol was placed within the wound cavity, with a $400-600 \mu \mathrm{l}$ pore size, cut and contoured to fit the size of the defect. The sponge was covered with an adhesive drape to obtain a sealed environment. (figure 3 and 4) Between the drape and the device, an electrical pump is connected to a canister that collects the wound exudate using a flexible pipe. Dressing is changed after 72 hours and post VAC culture is taken. These cycles of dressings and vacuum are applied and statistical assessment was done using outcome variables. VAC therapy is based on the standard surgical practice of vacuum-assisted drainage to remove blood or serous fluid from a wound or operation site. The negative pressure reduces the size of the cavity and facilitates fluid removal. The application of foam or gauze ensures that the negative pressure effect is uniformly distributed within the wound cavity, eliminating the possibility of localised areas of high pressure and possible resultant tissue necrosis or catastrophic haemorrhage. Furthermore, the use of foam or gauze prevents occlusion of the drain perforations because they are not in contact with the base or edges of the wound. The container that collects the wound exudate is changed weekly or upon reaching full capacity.

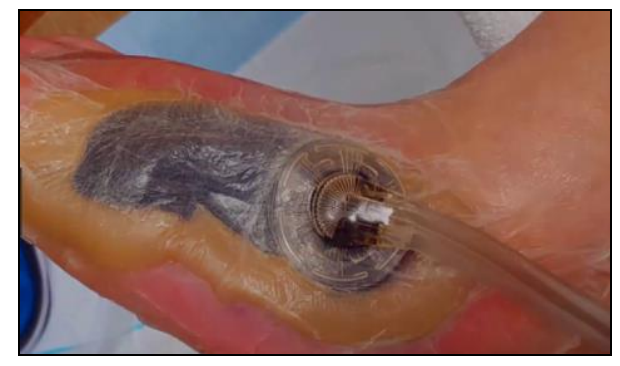

Fig 3: Application of VAC on foot

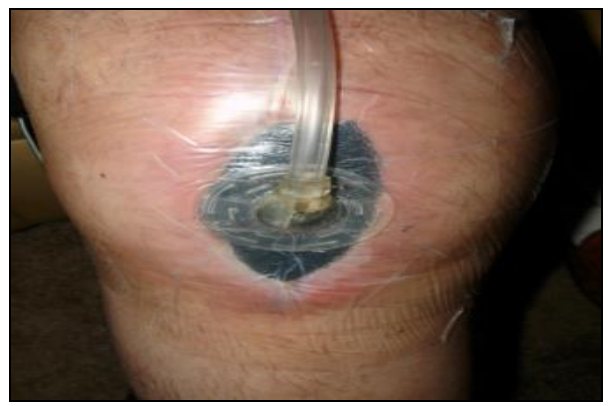

Fig 4: Application of VAC on knee

\section{Observation and Results}

The present prospective study comprises of 25 cases of post operative wound dehiscence that were eligible for inclusion during the period from 2016-2017. Our study confirms that vacuum assisted closure is an excellent treatment modality for post operative wound dehiscence. It is seen in elder age group were mainly due to associated comorbidity, mainly diabetes and smoking. We took x rays for all patients. Anteroposterior and lateral view. We have found it is very much informative to rule out osteomyelitis changes. We classified all wound according to Wagner classification, which we have found best. Since it gives idea about pathophysiology of wound and guideline for further treatment plan. Further it is simple with less inter-observer variation. The VAC dressing was changed every 3-4 days. Patients had an average of 8 (range 4-12) treatment sessions. The duration of VAC application ranged from 12 to 36 days and the treatment was stopped only after sufficient granulation tissue developed and in absence of local and systemic signs of infection, with an average hospital stay of 24 days. None of the patients reported pain or discomfort associated with the VAC system or noticed any odour associated with the VAC dressing. There were no reports of bleeding associated with the use of the system.

The portable VAC systems allowed patients to be ambulatory and facilitated early rehabilitation. During a mean follow-up of 12 months, we observed complete wound healing by secondary intention in 12 cases (48\% table 5), in an average period of 24 days. (Figure 6)

\section{Age Distribution}

The incidence of cases in various age groups is shown in table:

Table 1: Age Distribution ( $\mathrm{n}=25)$

\begin{tabular}{|c|c|}
\hline Age (years) & Subjects \\
\hline $21-30$ & $3(12 \%)$ \\
\hline $31-40$ & $5(20 \%)$ \\
\hline $41-50$ & $5(20 \%)$ \\
\hline $51-60$ & $6(24 \%)$ \\
\hline $61-70$ & $6(24 \%)$ \\
\hline
\end{tabular}

As seen in table 1, all patients are ranged from 21-70 years of age. Amongst this maximum patients being in the age group of 30-70 (88\%) years.

\section{Sex Distribution}

Table 2: Sex Distribution $(\mathrm{n}=42)$

\begin{tabular}{|c|c|}
\hline Sex & Subjects \\
\hline Male & $15(60 \%)$ \\
\hline Female & $10(40 \%)$ \\
\hline Total & 25 \\
\hline
\end{tabular}

In our study, there were $15(60 \%)$ male and $10(40 \%)$ female patients were present.

\section{Type of Wound}

Table 3: Classification of wound

\begin{tabular}{|c|c|}
\hline Wagner type & Subjects \\
\hline Grade 1 & $7(28 \%)$ \\
\hline Grade 2 & $8(32 \%)$ \\
\hline Grade 3 & $6(24 \%)$ \\
\hline Grade 4 & $4(16 \%)$ \\
\hline
\end{tabular}

\section{Duration of Hospital Stay}

Table 4: Duration of hospital stay

\begin{tabular}{|c|c|}
\hline duration of hospital stay in days & Subjects \\
\hline $7-14$ & 4 \\
\hline $14-21$ & 7 \\
\hline $21-28$ & 7 \\
\hline $28-35$ & 6 \\
\hline$>35$ & 1 \\
\hline
\end{tabular}


Duration of hospital stay was found to be statistically significant. Majority (72\%) of cases left hospital within 4 weeks.

\section{Surgical Plan after Vac}

Table 5: Surgical outcome

\begin{tabular}{|c|c|}
\hline Outcome/Plan & Subjects \\
\hline Closure by Secondary intention/suture & $12(48 \%)$ \\
\hline Split skin graft & $10(40 \%)$ \\
\hline Flap surgery & $3(12 \%)$ \\
\hline
\end{tabular}

VAC dressing found to produce more secondary closure of wound and split skin graft, also reduced more invasive procedures like flap surgery and in worst case amputation.

\section{Culture Sterility in Pre-Vac and Post-Vac State}

Table 6: Analysis of Culture Sterility in Pre-VAC and Post-VAC State

\begin{tabular}{|c|c|c|}
\hline Culture Sterility in Cases & Pre-VAC & Post-VAC \\
\hline Sterile & $5(20.00 \%)$ & $23(92.00 \%)$ \\
\hline Non-sterile & $20(80.00 \%)$ & $2(8.00 \%)$ \\
\hline Total & 25 & 25 \\
\hline
\end{tabular}

Table 6 shows that Patients with sterile pre-VAC culture are turning into $2(8.00 \%)$ unsterile after VAC. But unsterile culture turns $23(92.00 \%)$ sterile after VAC.

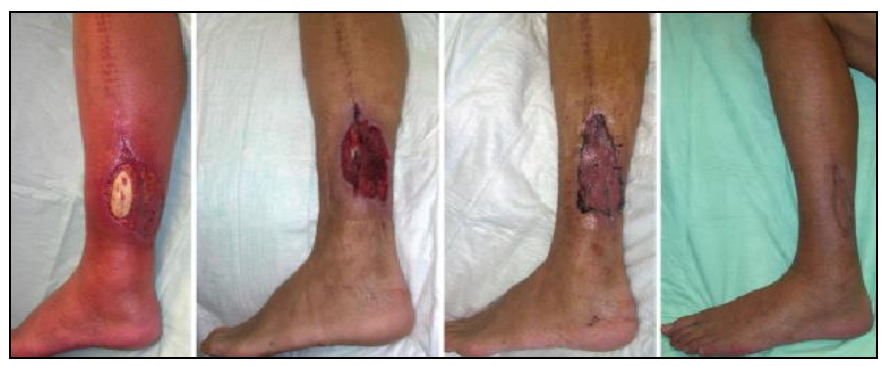

Fig 5: Wound management pre-VAC and post-VAC with skin grafting.

\section{Discussion}

The use of VAC is increasing, especially in reconstruction of complex wound defects. The VAC enhances the tissue granulation, which makes it possible to use less complex reconstruction options, e.g. converting the wounds acceptable for the skin grafting which otherwise would have required flap coverage ${ }^{[7]}$. Use of the suction tubing creates a continuous negative-pressure dressing. This device has been associated with accelerated development of granulation tissue. earlier re- epithelialization of wounds and faster healing of burn wounds and has been used to manage very complex wounds successfully.

Apart from decreasing hospital stay VAC dressing improves pus culture sterility, improves outcome by decreasing the number of amputations and increasing the number of patients undergoing flap surgery ${ }^{[8]}$.

Skin-graft take is dependent on immobilisation on a vascular bed. Various means of securing the skin have been used, including sutures and staples. The mode of action of the VAC machine has been well described and its use in securing splitthickness skin grafts has also been established ${ }^{[9]}$.

The VAC machine is particularly useful in the management of the wound dehiscence because it provides a constant conforming pressure to the replaced skin, allowing secure contact with the bed and potentially increasing the take of the graft in this difficult area by reducing haematoma and seroma formation ${ }^{[10]}$. In addition, it is easy to apply and nurse postoperatively. Reports of such patients receiving VAC therapy have been very encouraging and it may be that with the use of this machine a better reconstruction can be achieved than would be obtained by skin grafting using conventional dressings or by free-tissue transfer ${ }^{[11]}$. This raises the possibility of being able to restore a more normal gait than would be possible using a free flap and the technique has the potential advantages of reduced donor site morbidity, reduced length of hospital stay, reduced cost and earlier mobilisation. The time to mobilisation was particularly impressive in our patient, who commenced mobilisation with 4 weeks after his injury, and returned to independent living. Although most studies were probably too small to detect significant differences, some did show VAC to result in better healing than standard methods, with few serious complications ${ }^{[12]}$. VAC appears to be a promising alternative for management of wounds. VAC therapy appeared to be more effective than Opsite and bolster dressings in skin graft management ${ }^{[13]}$. VAC was also more effective at treating various chronic and complex wounds than WM gauze, with a significantly greater reduction in wound volume, depth and treatment duration for VAC ${ }^{[14]}$. Foot ulcers managed with VAC significantly decreased in surface area compared with those managed with saline moistened gauze; however, this is relying on data from a small pilot study ${ }^{[15]}$.

The treatment of post operative wounds suggests that VAC may be more cost-effective than traditional dressings, as VAC required a reduced number of dressing changes and number of flaps to close the wound, and a shorter treatment duration and length of hospital stay. This has the potential to reduce health care costs, for both hospital and patient, and enhance patient satisfaction and quality of life ${ }^{[16]}$.

A list of possible complications has been outlined by the manufacturer:

- Pain and discomfort when suction is applied initially (this usually resolves as therapy continues).

- Bleeding.

- Allergies to the adhesive drape.

- Excoriation (chafing) of the skin if foam is not correctly cut to size.

- Fulminant or incipient skin necrosis.

Although the studies included in this review did not report many of these types of complications, patients should be monitored to prevent discomfort.

\section{Advantages of VAC therapy Include}

- Reduced frequency of dressing changes, thus reducing nursing time for wound care and increasing patient comfort

- Reduced hospital length of stay

- Availability of portable VAC devices.

- Reduced bacterial cell count

- Enhanced dermal perfusion (blood flow to the wound).

- Removal of interstitial fluid to allow tissue decompression.

- Provision of a closed, moist wound healing environment.

It is imperative that patients and/or their care takers are taught to manage the system independently to ensure maximum effectiveness and safety. Although VAC is simple to apply, 
inappropriate and incorrect use will result in a non-healing wound and pain and discomfort to the patient. For example, active negative pressure therapy should be maintained for 22 to 24 hours per day and if therapy is turned off for longer than two hours, the dressing must be removed and replaced with a traditional dressing. It is also recommended that patients receive regular clinical evaluations to monitor progress and a nutritional evaluation to ensure adequate nutritional status.

Vacuum therapy is a useful method of temporarily covering soft tissue between initial surgery, second-look procedures, and definitive wound closure or definitive plastic surgery. A variety of older and more recent studies have reported good results with this technique, which are attributable to different mechanisms [17]. Compared with conventional methods, vacuum therapy has, in particular, been shown to improve bacterial clearance, increase local blood flow, and promote the formation of granulation tissue. Vacuum therapy has been found to be effective, not only in routine clinical practice, but also in the management of large numbers of patients with heavily contaminated soft tissue injuries. Good results were reported by Wu SH et al. ${ }^{[18]}$ in a study of 179 patients who had sustained severe open tibial and fibular fractures and massive soft tissue injuries during an earthquake and had been treated with external fixation and vacuum therapy. Positive results were also reported when vacuum therapy was used for the management of wounds in military conflicts. Compared with the injury patterns prevalent in European emergency departments, military wounds are far more often high-energy or blast injuries and associated with extensive soft tissue injuries and massive contamination. Two studies, both published by the US military ${ }^{[19]}$, retrospectively compared vacuum therapy to historical experiences and found that the use of vacuum therapy led to improved rates of healing, reduced levels of infection, and improved qualitative status of the wounds, providing an environment favourable for definitive wound closure. Similar results were reported by Mendonca DA et al. ${ }^{[20]}$ These investigators concluded that vacuum therapy cannot replace current wound management strategies such as debridement, fracture

stabilization, or definitive wound closure but that it can serve as a method of temporizing wounds and can thus provide time during which patients can be physiologically stabilized and other injuries can be addressed. Moreover, Morykwas MJ et al. ${ }^{[21]}$ found lower rates of necessary amputations than those reported during previous military conflicts and attributed a large part of this success to the use of vacuum-assisted wound closure.

These results are in line with those from other studies confirming the integral role of vacuum therapy in the initial management of single or multiple injuries and extensive soft tissue defects with and without bone involvement. In a recent study of 36 patients with large soft tissue injuries involving exposure of lower limb bone, Freundlich BD et al. ${ }^{[22]}$ found that vacuum therapy was not only effective in promoting the growth of granulation tissue in the wound bed and in temporarily covering the wound but also helped avoid lower limb amputation and complex surgery.

Just as in our present case, an extensive open injury cannot be closed primarily.

The options for the initial management of this injury pattern are thus limited. Plastic surgical procedures for the coverage of open wounds usually require wound that is free of infection. In addition, degloving injuries require a critical inspection of the blood supply to the affected region and can necessitate microsurgical revascularization. Although plastic surgical procedures can achieve safe and stable wound closure, the absence of nerve sensations can lead to secondary problems, including gait unsteadiness and pressure ulcers ${ }^{[23]}$. The sealing of a wound with a vacuum dressing system allows the wound area to be covered in a sterile fashion and thus helps to prevent further contamination. Ravari et al. [24] reported a significant reduction in the diameter and depth of wounds in patients with diabetic foot ulcers who received vacuum-assisted closure compared with patients who received conventional dressings. Direct closure of some wound sites could thus be possible during the course of treatment. Other wound sites can benefit from the local increase in blood flow and the promotion of granulation tissue formation. In these cases, vacuum therapy can be used to enhance wound bed preparation before secondary plastic surgery. Appropriate initial wound management is of particular relevance if the graft recipient site will be subject to high stresses and strains and if the restoration of function is of paramount importance, such as in the present case. The successful management of a degloving injury using vacuum therapy described previously by Josty IC ${ }^{[25]}$ underlines our findings that this therapy can be regarded as an effective and safe treatment option in the management of these injury patterns. In the present case, VAC achieved an excellent functional outcome, which was particularly important because of the demanding requirements that a foot must meet. In addition to these complex procedures in managing soft tissue injuries, the treating surgeon should always be aware of the potential for the development of compartment syndrome. Even as an open

fracture and/or a skin avulsion is treated, high pressures in the deep muscle compartments can develop after this type of trauma, and fasciotomies will be required as additional treatment.

\section{Conclusion}

This study shows a positive effect of vacuum assisted closure therapy on wound healing, expressed as a significant reduction in hospital stay subsequent lower in hospital cost, decreased amputations, increasing the number of patients undergoing skin grafting and improving culture sterility. It also promotes early rehabilitation, and alleviates the need for a second procedure, thus improving patient satisfaction with minimal discomfort or inconvenience. The application of $\mathrm{VAC}$ is simple, but requires training to ensure appropriate and competent use. From our observations, we support using negative-pressure dressings over post operative wound dehiscence and believe that to better quantify outcome measures, further study of this device in a prospective, randomized fashion is warranted.

\section{Conflicts of Interest}

There are no conflicts of interest.

\section{Acknowledgement}

We would like to acknowledge residents of Orthopaedics, radiology and pathology for contributing for this case series. Also lab technicians whose sincere effort made this case report possible.

\section{References}

1. Bovill E, Banwell PE, Teot L, Eriksson E, Song C, Mahoney $\mathrm{J}$ et al. International Advisory Panel on Topical Negative Pressure. Topical negative pressure wound therapy: a review of its role and guidelines for its use in the management of acute wounds. Int Wound J. 2008; 
5:511-29.

2. Fleischmann W, Strecker W, Bombelli M, Kinzl L. Vacuum sealing as treatment of soft tissue damage in open fractures. Unfallchirurg. 1993; 96:488-92.

3. Argenta LC. Vacuum device can speed healing. Wound Care, 1997, 29.

4. Müllner T, Mrkonjic L, Kwasny O, Vécsei V. The use of negative pressure to promote the healing of tissue defects: a clinical trial using the vacuum sealing technique. Br J Plast Surg. 1997; 50:194-9.

5. Blackburn JH, Boemi L, Hall WW, Jeffords K, Hauck RM, Banducci DR. Graham WP 3rd. Negative-pressure dressings as a bolster for skin grafts. Ann Plast Surg. 1998; 40:453-7.

6. De Caridi G, Massara M, Greco M, Pipitò N, Spinelli F, Grande R et al. VAC therapy to promote wound healing after surgical revascularisation for critical lower limb ischaemia. Int. Wound J. 2014. doi: 10.1111/iwj.12301.

7. Morykwas MJ, Faler BJ, Pearce DJ, Argenta LC. Effects of varying levels of subatmospheric pressure on the rate of granulation tissue formation in experimental wounds in swine. Ann Plast Surg. 2001; 47:547-51.

8. DeFranzo AJ, Marks MW, Argenta LC, Genecov DG. Vacuum assisted closure for the treatment of degloving injuries. Plast Reconstr Surg. 1999; 104:2145-8.

9. Argenta LC, Morykwas MJ. Vacuum-assisted closure: a new method for wound control and treatment: clinical experience. Ann Plast Surg. 1997; 38:563-76.

10. Wong LK, Nesbit RD, Turner LA, Sar-Gent LA. Management of a circumferential lower extremity degloving injury with the use of vacuum-assisted closure. South Med. J. 2006; 99(6):628-30.

11. Defranzo AJ, Marks MW, Argenta LC. Vacuum-assisted closure for the treatment of degloving injuries. Plast. Reconstr. Surg. 1999; 104:2145-2148.

12. Meara JG, Guo L, Smith JD. Vacuum-assisted closure in the treatment of degloving injuries. Ann. Plast. Surg. 1999; 42:589-594.

13. Wysocki AB, Grinnell F. Fibronectin profiles in normal and chronic wound fluid. Lab. Invest. 1990; 63:825.

14. Falanga V. Chronic wounds: Pathophysiologic and experimental considerations. J Invest Dermatol. 1993; 100:721.

15. Heller L, Levin SL, Butler CE. Management of abdominal wound dehiscence using vacuum assisted closure in patients with compromised healing. Am J Surg. 2006; 191(2):165-72.

16. Wysocki AB, Staiano-Coico L, Grinnell F. Wound fluid from chronic leg ulcers contains elevated levels of metalloproteinases MMP-2 and MMP-9. J Invest. Dermatol. 1993; 101:64.

17. De Franzo AJ, Argenta LC, Marks MW. The use of vacuum assisted closure therapy for the treatment of lower extremity wounds with exposed bone. Plast Reconstr Surg. 2001; 108(5):1184-91.

18. Wu SH, Zecha PJ, Feitz R. Vacuum therapy as an intermediate phase in wound closure: A clinical experience. Eur J Plast Surg. 2003; 23:174-6.

19. Van Der Velde M, Hudson DA. Vader (vacuum assisted dermal recruitment): a new method of wound closure. Ann Plast Surg. 2005; 55(6):660-4.

20. Mendonca DA, Cosker T, Makmana NK. Vacuumassisted closure to aid wound healing in foot and ankle surgery. Foot Ankle Intl. 2005; 26(9):761-6.

21. Morykwas MJ, Argenta LC, Shelton-Brown EI. Vacuum- assisted closure: a new method for wound control and treatment: animal studies and basic foundation. Ann Plast Surg. 1997; 38(6):553-62.

22. Freundlich BD, Dashiff JE. Avulsion of tibialis anticus and peronei muscles resulting in acute anterior and lateral compartment syndrome. J Trauma. 1987; 27:453-454.

23. Shadgan B, Pereira G, Menon M, Jafari S, Darlene ReidW, O'Brien PJ. Risk factors for acute compartment syndrome of the leg associated with tibial diaphyseal fractures in adults. J Orthop Traumatol. 2015; 16:185192.

24. Ravari H, Modaghegh MHS, Kazemzadeh GH, Johari HG, Vatanchi AM, Sangaki A et al. Comparison of vacuum-assisted closure and moist wound dressing in the treatment of diabetic foot ulcers. J Cutan Aesthet Surg. 2013; 6:17-20.

25. Josty IC, Ramaswamy R, Laing JH. Vacuum assisted closure: an alternative strategy in the management of degloving injuries of the foot. Br J Plast Surg. 2001; 54:363-365. 